Vaccine delivers neutralization

\title{
$A$ vaccine that delivers rather than induces antibodies
}

NL Letvin

Gene Therapy (2009) 16, 1283-1284; doi:10.1038/gt.2009.121; published online 17 September 2009

Protection against an AIDS virus infection in monkeys has recently been described using a novel strategy for vaccination-the use of the adeno-associated virus (AAV) as a gene transfer vector to initiate the persistent in vivo production of a single-chain antibody-like molecule.

Most vaccines confer protection against virus infections by stimulating the induction of memory B cells and plasma cells that produce antibodies that bind to the virus and block the ability of that virus to infect cells. The production of this type of antibodies-referred to as neutralizing antibodies-is triggered by inoculating an individual with altered virus or viral protein. This approach to generating antibody production has been the basis for effective vaccines against a diversity of viruses, including poliovirus, hepatitis B virus and influenza.

This strategy, however, has not proven useful for preventing infection with human immunodeficiency virus (HIV). One of the reasons for the ineffectiveness of this vaccine strategy is a consequence of the extreme genetic diversity of HIV. There is such sequence diversity of HIV that an antibody with the ability to neutralize one isolate of the virus might not neutralize another.

Motivated by the failure of traditional vaccine strategies for preventing HIV infection, Johnson et al. ${ }^{1}$ have examined a radically different approach to inducing long-term production of neutralizing antibodies against an AIDS virus. They used AAV to deliver, directly into the muscles of monkeys, a gene that encodes a single-chain antibody-like molecule that neutralizes the monkey AIDS virus. High titer neutralization was detected in the serum of most monkeys within days after inoculation with this recombinant
AAV construct. Although all six monkeys that did not receive the experimental vaccine were infected after virus challenge, six of the nine monkeys that received the experimental vaccine were protected from infection.

This finding has generated excitement in the HIV vaccine-development community, and for good reason. The vaccine prototypes that have been tested for protection against HIV infection in human volunteers have shown no evidence of efficacy. Even the most promising of the novel AIDS virus vaccine prototypes that have been evaluated to date in monkeys confer sufficient immunity to blunt virus replication, but not sufficient immunity to prevent acquisition of virus. $^{2,3}$ The radical strategy for vaccination against HIV described by Johnson et al. ${ }^{1}$ therefore warrants further exploration.

However, two substantial hurdles must be overcome to make this approach viable for use as an AIDS vaccine. First, this strategy faces a problem well known to the gene therapy community-the induction of immunity against the product of the transgene introduced by the vector. Antibodies were generated in the experimentally vaccinated monkeys against the antibody-like molecules produced by the transgene. Importantly, these induced antibodies blocked the efficacy of the antibodylike molecules produced in vivo by the recombinant $\mathrm{AAV}$. In fact, the absence of protection against an AIDS virus infection in three of the nine experimentally vaccinated monkeys could be linked to the presence of high levels of circulating antibodies against the antibody-like molecules in these animals.

This problem may have a solution. Therapeutic successes have been reported associated with the admin- istration of monoclonal antibodies that neutralize viruses, inactivate cytokines or eliminate discrete, harmful populations of cells. As repeated delivery of such monoclonal antibodies induces antibody responses that block the efficacy of those monoclonal antibodies, there is considerable work ongoing to 'de-immunize' these infused monoclonal antibodies. ${ }^{4-6}$ The experience gained in engineering monoclonal antibodies to diminish their immunogenicity should inform attempts to create novel antibody-like molecules that can neutralize HIV, but not induce immune responses that block the therapeutic efficacy of the molecules.

The second problem standing in the way of using this strategy for the creation of an AIDS vaccine may prove more daunting. Despite considerable effort by a large number of laboratories, very few monoclonal antibodies have been generated that neutralize a diversity of HIV isolates. Even the few antibodies that neutralize a variety of HIV isolates only neutralize those isolates obtained from geographically circumscribed regions of the world. ${ }^{7}$ Unless monoclonal antibodies can be generated that neutralize a substantial percentage of HIV isolates present in human populations worldwide, this strategy for the creation of an AIDS vaccine will not prove effective.

In spite of these caveats, the recently reported findings of Johnson et al. represent a provocative approach to a vexing problem. Even if this strategy cannot be used for generating sterilizing immunity against HIV, it may find other vaccine or therapeutic applications.

\section{Conflict of interest}

The author declares no conflict of interest.

Professor NL Letvin is at Beth Israel Deaconess Medical Center, Harvard Medical School, Harvard University, 330 Brookline Avenue, Boston, Massachusetts 02115, USA.

E-mail:nletvin@bidmc.harvard.edu

1 Johnson PR, Schnepp BC, Zhang J, Connell MJ, Greene SM, Yuste E et al. Vector-mediated gene transfer engenders long-lived neutralizing activity and protection against SIV infection in monkeys. Nat Med 2009; 15: 901-906. 
2 Letvin NL, Mascola JR, Sun Y, Gorgone DA, Buzby AP, Xu L et al. Preserved CD4(+) central memory $T$ cells and survival in vaccinated SIV-challenged monkeys. Science 2006; 5779: 1530-1533.

3 Liu JY, O'Brien KL, Lynch DM, Simmons NL, La Porte A, Riggs AM et al. Immune control of an SIV challenge by a T-cell-based vaccine in rhesus monkeys. Nature 2009; 7225: 87-91.
4 Tangri S, Mothe BR, Eisenbraun J, Sidney J, Southwood S, Briggs $\mathrm{K}$ et al. Rationally engineered therapeutic proteins with reduced immunogenicity. J Immunol 2005; 174: 3187-3196.

5 Perry LCA, Jones TD, Baker MP. New approaches to prediction of immune responses to therapeutic proteins during preclinical development. Drugs RED 2008; 9: 385-396.
6 Holgate RGE, Baker MP. Circumventing immunogenicity in the development of therapeutic antibodies. IDrugs 2009; 12: 233-237.

7 Binley JM, Wrin T, Korber B, Zwick MB, Wang $\mathrm{M}$, Chappey $\mathrm{C}$ et al. Comprehensive cross-clade neutralization analysis of a panel of anti-human immunodeficiency virus type 1 monoclonal antibodies. J Virol 2004; 78: 13232-13252. 